\title{
Evaluation of Chemical Exposures during Optical Media Production
}

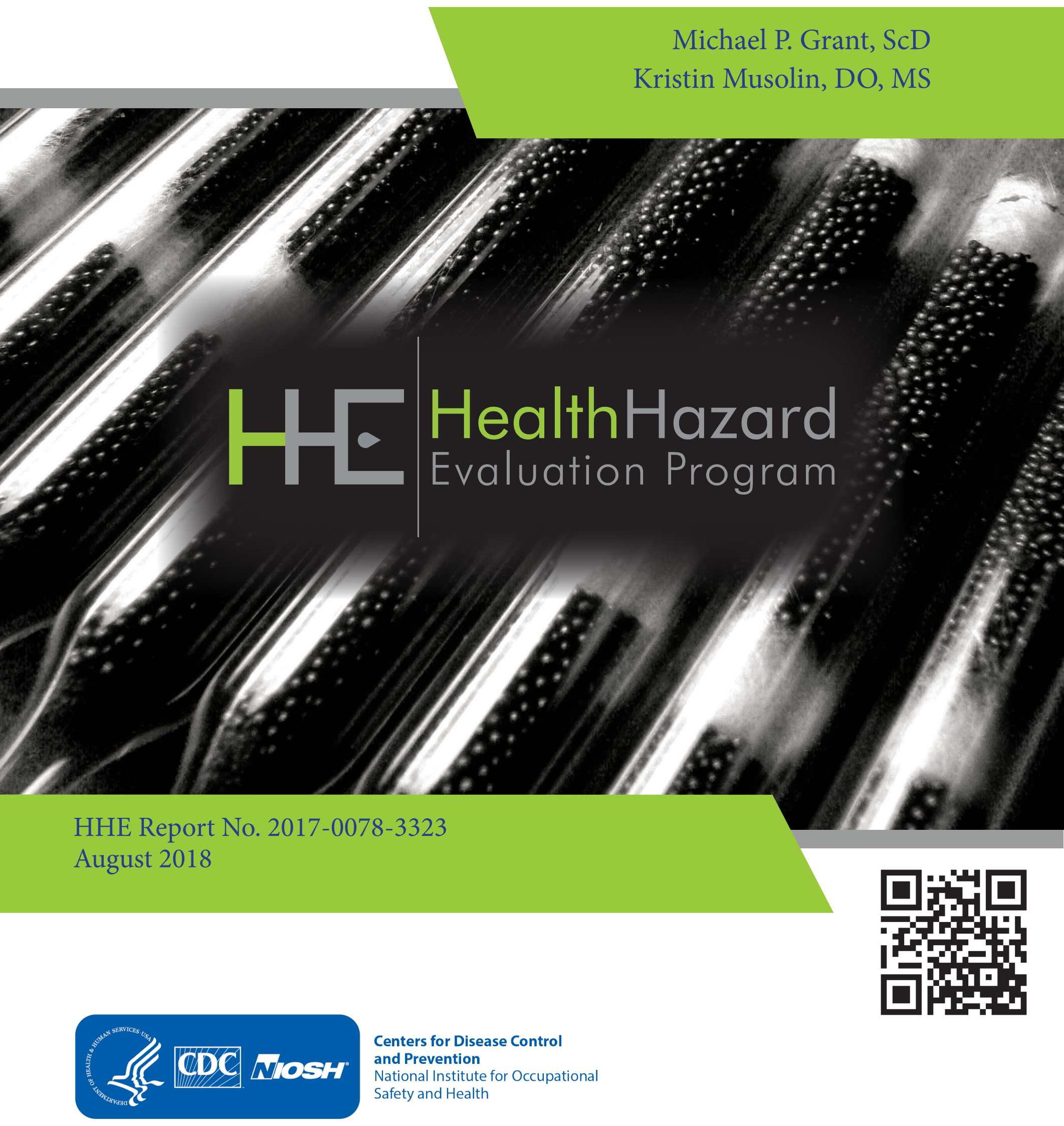




\section{Contents}

Highlights.

Abbreviations ...................................... iii

Introduction ....................................... 1

Methods ............................................. 2

Results and Discussion ........................ 3

Conclusions........................................... 12

Recommendations............................. 12

Appendix A ......................................... 16

References........................................... 18

Acknowledgements........................... 21

The employer is required to post a copy of this report for 30 days at or near the workplace(s) of affected employees. The employer must take steps to ensure that the posted report is not altered, defaced, or covered by other material.

The cover photo is a close-up image of sorbent tubes, which are used by the HHE Program to measure airborne exposures. This photo is an artistic representation that may not be related to this Health Hazard Evaluation. Photo by NIOSH. 


\section{Highlights of this Evaluation}

The Health Hazard Evaluation Program received a request from an employer at an optical media production company. The request concerned chemical odors associated with optical media production. We visited the company in September 2017.

\section{What We Did}

- We observed employees while they manufactured optical media.

- We collected air samples from employees and in different areas over two shifts.

- We sampled for ozone at the ultraviolet curing exhaust for the molding machines.

- We visually inspected the accessible sections of the ventilation system serving the molding and printing departments.

- We assessed airflow in the molding and printing departments as well as on the rooftop.

- We measured the face velocity of the screen wash hood in the printing department.

- We asked employees about their work history, chemical exposure, personal protective equipment, work-related symptoms, and health and safety concerns.

- We reviewed incident report logs for the years 2013-2017.

- We reviewed one employee's medical record.

- We reviewed safety data sheets.

We evaluated chemical exposures during optical media production. We did not detect naphthalene, ethylbenzene, xylene, 2-butoxyethanol, or trimethylbenzene in any of the full-shift air samples. Hazard communication needed improvement and some employees reported symptoms in the last 6 months that may be due to chemical exposure or other causes. We recommended improving hazard communication and forming a health and safety committee.

\section{What We Found}

- We did not detect naphthalene, ethylbenzene, xylene, 2-butoxyethanol, or trimethylbenzene in any of the full-shift air samples. We did detect a variety of volatile organic compounds in short-term screening samples. There was no ozone detected at the ultraviolet curing exhaust for the molding machines in use during our visit.

- Hazard communication needed improvement. Safety data sheets were not up to date and employees reported not receiving training related to the hazards of work and strategies for protecting themselves while at work.

- Many of the employees who worked with solvents reported occasionally noticing solvents on uncovered parts of their body, especially their arms, hands, and/or face.

- Most commonly reported symptoms by employees were lightheadedness, headache, and dizziness in the last 6 months. 
- Many employees reported a transient unpleasant odor or taste in mouth in the last 6 months.

\section{What the Employer Can Do}

- Form a health and safety committee or working group to discuss our recommendations and develop an action plan.

- Replace the handmade hood with one that complies with the appropriate standards.

- Consult with a ventilation engineer to further investigate the source of, and to identify a way to prevent liquid from forming in the ductwork between the molding and printing departments.

- Improve hazard communication within the workplace.

- Replace latex gloves with thick nitrile gloves.

- Require safety glasses be worn by all employees, contractors (i.e., cleaning contractors, temporary agency staff), and visitors in the molding and printing departments.

- Prohibit food and drink inside the molding and printing departments.

- Encourage all employees in the molding and printing departments to wear nitrile gloves and long sleeves to prevent unnecessary dermal exposures to solvents.

- Encourage all employees to promptly report work-related health and safety concerns.

\section{What Employees Can Do}

- Do not eat or drink inside the molding and printing departments.

- Wash your hands and exposed areas of the body with water and soap after removing gloves and before eating, smoking, or using the restroom.

- Follow all relevant health and safety requirements.

- Tell your supervisor about symptoms that you believe are work-related. If symptoms persist, see a healthcare provider who is knowledgeable in occupational medicine.

- Wear nitrile gloves and long sleeves to prevent unnecessary dermal exposures to solvents. 


\section{Abbreviations}

ACGIH ${ }^{\circledR} \quad$ American Conference of Governmental Industrial Hygienists

ANSI

American National Standards Institute

CFR

Code of Federal Regulations

$\mathrm{NIOSH}$

National Institute for Occupational Safety and Health

OEL

Occupational exposure limit

OSHA

Occupational Safety and Health Administration

PPE

Personal protective equipment

SDS

Safety data sheet

TLV®

Threshold limit value

TWA

Time-weighted average

VOC

Volatile organic compounds 
This page left intentionally blank 


\section{Introduction}

The National Institute for Occupational Safety and Health (NIOSH) Health Hazard Evaluation Program received a request from an employer at an optical media production company. The request concerned chemical odors associated with optical media production. In September 2017, we visited the company to evaluate the optical media production process. We evaluated employee exposures to volatile organic compounds (VOCs); spoke with managers and employees; and observed workplace conditions, processes, and practices. We provided our preliminary recommendations in a letter to the employer and the employee representative in September 2017.

The company was opened in 1984 and manufactured optical media (compact discs and digital video discs) using primarily resin, lacquer, ink, and screen wash chemicals. Employees work an 8-hour first- or second-shift, Monday through Friday and occasionally on Saturday. Additional shifts or days of work may be scheduled depending on the workload and requirements of specific orders. The building had two floors and covered approximately 57,000 square feet including an attached warehouse. We focused our evaluation on six departments within the company: molding, printing, production, information technology, fulfillment, and administrative.

\section{Production Department}

In the production department, employees' primary duties included assembly of products (e.g., compact/digital video discs), stocking the tables with products, and/or packaging and shipping preparation. Employees in this department work first- and second-shift, but have a smaller number of employees working second-shift. This department is across the hall from the molding and printing departments and adjacent to the fulfillment warehouse.

\section{Molding Department}

There are four disc molding machines in the molding department. Employees' primary duties included setting/cleaning up (e.g., ensuring proper functioning of molding equipment and machinery, cleaning barrels, stampers and machines; removing lacquer from machine parts, following machine-specific procedures for purging and repairing mechanical parts), and/ or checking quality control of the products. Employees in this department work first- and second-shift, but have a smaller number of employees working second-shift. This department is adjacent to the printing department.

\section{Printing Department}

There are three printing machines in the downstairs printing area. There is one printing machine in the upstairs printing area. Employees' primary duties included setting/cleaning up (e.g., ensuring proper functioning of printing equipment and machinery, loading and unloading of discs, replacing screens and ink, and/or washing screens). Employees in this department work first-shift. This department is adjacent to the molding department and the fulfillment warehouse. 


\section{Information Technology Department}

In the information technology department, employees' primary duties included maintaining and managing computer systems (e.g., programming, coding, and switching out computers). Employees in this department work first-shift. This department is on the second floor directly above the molding department.

\section{Administrative Department}

In the administrative department, employees' primary duties included fielding questions and interacting with customers. Employees in this department work first-shift. This department is located on the first floor in a separate wing from the other departments.

\section{Fulfillment Department}

In the fulfillment department, employees' primary duties included packing orders for customers and loading/unloading delivery trucks. Employees in this department work first-shift. This department works mainly in the warehouse that is adjacent to the printing and production areas.

\section{Methods}

The objective of this project was to characterize exposures associated with optical media production. To accomplish this objective we (1) evaluated sources of airborne exposures to VOCs in the production, molding, printing, information technology, administrative, and fulfillment departments; (2) investigated the ultraviolet curing process and whether it produced ozone during optical media production; (3) evaluated the ventilation system; (4) determined whether employees were experiencing symptoms associated with VOC exposure; and (5) determined employees' health and safety concerns.

\section{Volatile Organic Compounds}

The most commonly used chemicals in the molding and printing areas are a resin and lacquer used in the molding department and ink and screen wash in the printing department. Prior to the visit, we reviewed safety data sheets (SDS) for commonly used chemicals at the company. We determined target analytes based on frequency of use, likelihood of exposure, and availability of an appropriate analytical method.

We collected personal air samples from employees in the production, molding, printing, administrative, and information technology departments. We collected both full-shift and short-term samples. The full-shift personal air samples were analyzed for naphthalene, ethylbenzene, xylene, 2-butoxyethanol, and trimethylbenzene according to NIOSH Method 1403, 1501, and 1550 [NIOSH 2018]. The short-term air samples were intended to screen for additional VOCs that were not major components of the chemicals used in the optical media production process. The short-term personal samples were qualitatively screened for VOCs using thermal desorption tubes according to NIOSH Method 2549 [NIOSH 2018]. We collected full-shift and short-term area air samples in the same departments as the personal air samples, with additional samples in the fulfillment department. All area samples were analyzed in the same manner as the personal air samples described previously. 


\section{Ozone}

We sampled air from the exhaust of the ultraviolet curing process using ozone tubes (Dräger) to see if we could detect ozone. We focused on the molding machines that were operational during our visit and sampled in the middle of a production run. We sampled for ozone while the three molding machines were in operation. We sampled a few hours into the second-shift. The machines had been in continuous operation for the majority of the day. Each sample used 10 strokes of air through the Dräger tube, which was held close to the exhaust location from the UV curing process.

\section{Ventilation}

We inspected the ventilation system at the company including the air handling units, a hood, and ductwork. We used qualitative and quantitative methods to characterize airflow in the molding and printing departments. We checked airflow direction at each doorway leading to the molding and printing departments to determine whether the departments were under positive or negative pressure relative to the production and fulfillment departments. We used ventilation smoke tubes (Dräger) to visualize airflow patterns. We used a Shortridge Instruments, Inc. AirData Multimeter Model ADM-860C electronic micromanometer to measure air velocity.

\section{Employee Medical Interviews}

We obtained a list of all employees on the first- and second-shift working in the production, molding, printing, and information technology departments and held confidential interviews in English or Spanish (based on employee preference) with those that were present during our visit. We asked about employees' work history, chemical exposure, personal protective equipment (PPE), work-related symptoms, and health and safety concerns.

\section{Document Review}

We reviewed the Worker's Compensation Incident Report Log for the period covering 2013-2017. We also reviewed medical records for one employee who visited healthcare providers because of symptoms possibly related to exposure to chemicals (e.g., VOCs) after manufacturing optical media. In addition, we asked to examine the last five years of their Occupational Safety and Health Administration (OSHA) Form 300 (Log of Work-Related Injuries and Illnesses), OSHA Form 300A (Summary of Work-Related Injuries and Illnesses), and OSHA Form 301 (Injury and Illness Incident Reports).

\section{Results and Discussion}

\section{Volatile Organic Compounds}

For employees working the first-shift, we collected full-shift personal air samples from 10 employees in five departments (molding, production, printing, administrative, and information technology) and area air samples in all six departments, for the targeted analytes. The personal air samples ranged from 164 to 512 minutes, averaging 390 minutes 
in duration. Area air samples ranged from 448 to 491 minutes, averaging 466 minutes in duration. For employees working the second-shift we collected full-shift personal air samples from two employees in two departments (molding and production) and area air samples in three departments (molding, production, and administrative). The personal air samples averaged 479 minutes in duration. Area air samples averaged 471 minutes in duration. We did not detect naphthalene, ethylbenzene, xylene, 2-butoxyethanol, or trimethylbenzene in any of the air samples collected. The minimum detectable concentration for naphthalene, xylene, 2-butoxyethanol, and trimethylbenzene ranged from 0.001 to 0.1 parts per million. For ethylbenzene the minimum detectable concentration ranged from 0.0006 to 0.01 parts per million. It is not surprising that we did not detect these chemicals as employees used the screen wash inside the hood and for short amounts of time. We observed a chemical smell when the screen wash was dispensed onto rags prior to being used to clean screens. The occupational exposure limits (OEL) for these compounds can be found in Table 1.

Table 1. Occupational exposure limits for selected VOCs (parts per million)

\begin{tabular}{lccc}
\hline Analyte & OSHA PEL & NIOSH REL & ACGIH® TLV® \\
\hline 2-Butoxyethanol & 50 & 5 & 20 \\
Ethylbenzene & 100 & 100 & 20 \\
Naphthalene & 10 & 10 & 10 \\
Trimethylbenzene & - & 25 & 25 \\
Xylene & 100 & 100 & 100 \\
\hline ACGIH TLV = American Conference of Governmental Industrial Hygienists Threshold Limit Value \\
PEL = Permissible exposure limit \\
REL = Recommended exposure limit
\end{tabular}

We collected short-term personal air samples from 10 employees working the first-shift in five departments (molding, production, printing, administrative, and information technology) and area air samples in all six departments, using thermal desorption tubes. The short-term personal air samples ranged from 82 to 94 minutes, averaging 88 minutes in duration. Short-term area air samples ranged from 79 to 81 minutes, averaging 80 minutes in duration. We collected shortterm personal air samples from two employees working the second shift in two departments (molding and production) and area air samples in three departments (molding, production, and administrative). The short-term personal air samples averaged 83 minutes in duration while the short-term area air samples averaged 85 minutes. We identified a variety of VOCs in the shortterm air samples including: solvents (i.e., acetone, 2-butoxyethanol, 2-butoxyethyl acetate, ethanol, isopropyl alcohol); aliphatic hydrocarbons (i.e., decane, heptane, hexane, octane); aromatic hydrocarbons (i.e., ethylbenzene, naphthalene, toluene, xylene); esters (i.e., ethyl acetate); chlorinated solvents (i.e., chlorobenzene); and terpenes (i.e., limonene). Because this is a qualitative method, it does not provide a concentration of these chemicals.

Some of the compounds identified in the short-term samples could be attributed to the screen wash chemicals used in the printing department. Identifying these compounds in the short-term air samples but not in the full-shift air samples is not surprising. The short-term air sampling 
method is very sensitive to extremely low levels of chemicals. Additionally, these chemicals are found in the screen wash commonly used in the printing department. Screen wash is used periodically throughout the shift in small amounts dispensed onto rags. In the downstairs printing area, the screen wash is stored and dispensed inside of the hood (Figure 1). In the upstairs printing area, the screen wash is stored and dispensed out in the open.

Many of the compounds that were identified in the air samples are common in indoor environments. The compounds can be released from a variety of building materials [Wallace 1986, 1991; Wallace et al. 1987]. Some common materials that could produce these and similar compounds are adhesives, ceiling tiles, carpeting, furniture, cleaning products and aerosol spray products. Additionally, personal care products like perfume and cologne, deodorants, nail polish, and hair spray can contain these compounds.

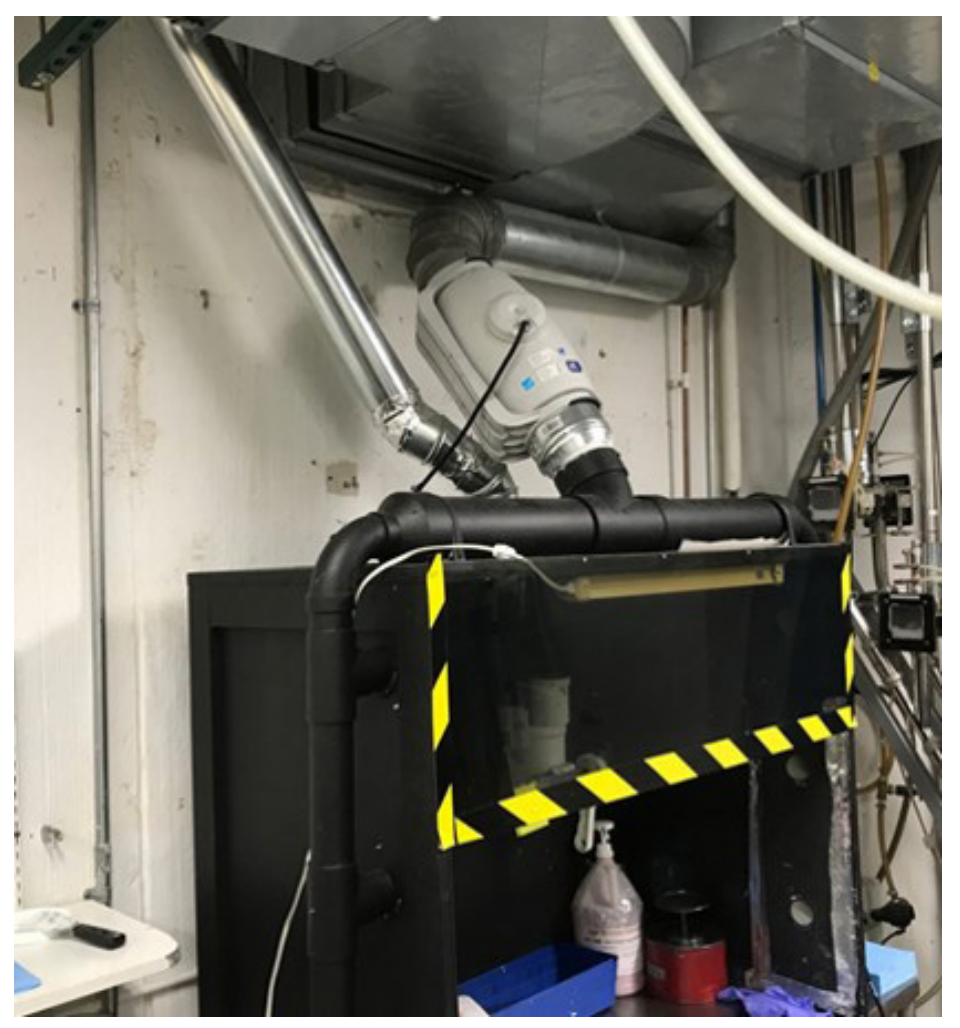

Figure 1. Photograph of the handmade hood in the printing department. Screen wash is dispensed from the red plunger can inside the hood, and the sash is not adjustable. Photo by NIOSH.

\section{Ozone}

There was no ozone detected at the ultraviolet curing exhaust for the three molding machines that were being used during our visit. The measuring range for ozone was 0.05 to 0.7 parts per million. All machines were also equipped with exhaust ventilation attached directly to the machine so ozone exposure is likely minimized. While the machines were not airtight, the exhaust appeared to be adequate to mitigate ozone produced during this process. These results support previous negative sampling results that the company requested from a consulting firm in 2014. 


\section{Ventilation}

The smoke tests revealed that air was negatively pressurized inside the molding and printing departments. That means that air moves from the hallways into the molding and printing departments. Airflow was also observed flowing from the warehouse into the printing department.

The molding department had three molding machines that created optical media discs. Each of the machines had an exhaust port attached directly to an exhaust ventilation system. Although the molding machines were not designed to be airtight, these connections were intended to capture the exhaust from the molding process. We used ventilation smoke around the machines. We detected slight air movement from the room into the cracks around the door of the machine when the machine was running with the doors closed. Between the molding and printing departments, the ductwork was raised vertically to accommodate the wall. There was a container and plastic tubing connected to the ductwork that drained and collected liquid (Figure 2). It is unclear where this liquid was coming from or how often the container was emptied. It is possible that it was condensation that occurred due to the temperature changes between the molding and printing department.

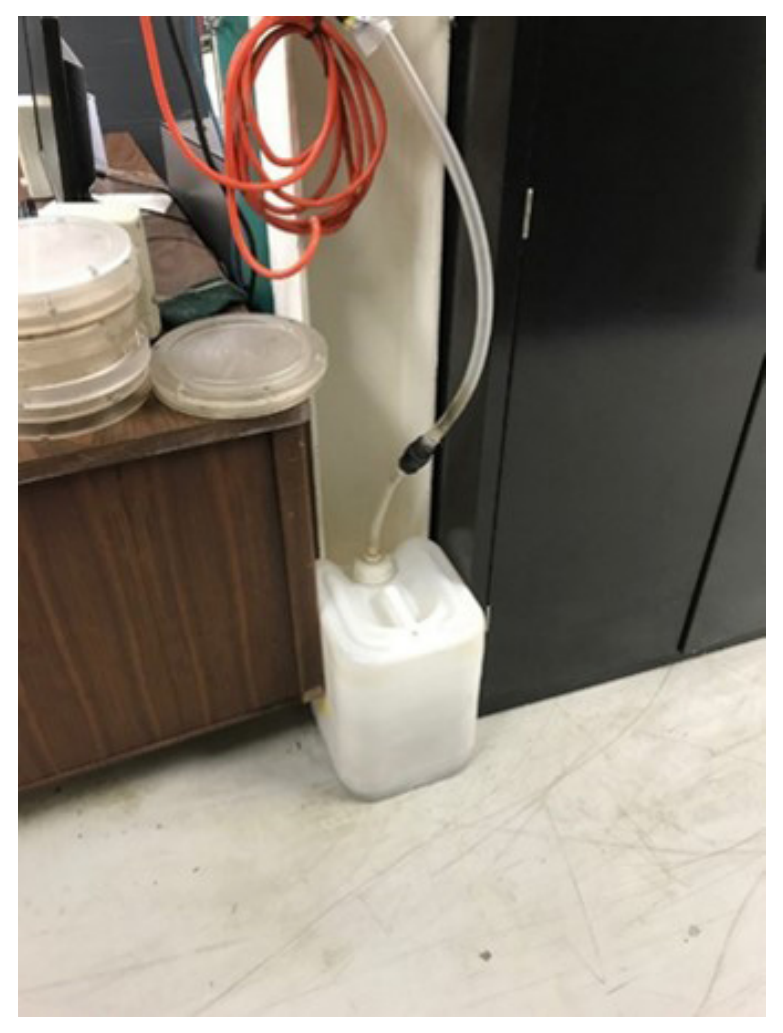

Figure 2. Catch basin that is connected to the ductwork between the molding and printing departments. There is a hose running up the wall connecting to the ductwork between the molding and printing departments (ductwork not pictured). Photo by NIOSH. 
The printing department used a hood when working with the screens used for printing (Figure 1). Employees removed the screen from the printer and brought it to the hood when it needed to be cleaned. The screens were cleaned with a screen wash chemical that was dispensed from a plunger can. Employees donned nitrile gloves, depressed the plunger three to five times with a disposable rag, and proceeded to clean the screen inside the hood. Rags were disposed of and rewetted with screen wash as necessary. In the downstairs printing area, there was a drying rack behind the sash where screens were stored until they were needed again. Upstairs, there was no hood. Employees in the upstairs printing area perform similar tasks without any ventilation beyond the general ventilation in the building.

The main housing of the hood in the printing department was constructed with wood and a plastic sash. The housing sits on top of a stainless steel table. There were two different ventilation systems that connected to the top of the hood. One system connected to the top of the main housing of the hood using a single four-inch duct. The other system exhausts air from the sides of the face of the hood in six locations, three on each side (Figure 3 ). The average face velocity was 46 feet per minute with non-uniform flow (Figure 4.). The Occupational Safety and Health Administration (OSHA) standard for occupational exposures to hazardous chemicals in laboratories [29 CFR 1910.1450] requires a "relatively uniform airflow" that is between 60 and 110 feet per minute. The American National Standards Institute (ANSI) Laboratory Ventilation Standard Z9.5 recommends an average face velocity between 80 and 120 feet per minute and no face velocity measurement more than $20 \%$ different from the average velocity. Based on our measurements, this hood does not meet the OSHA standard or the ANSI recommendations. Although the printing department was not a laboratory, these guidelines for face velocity are considered protective for workers handling chemicals in a fume hood. 


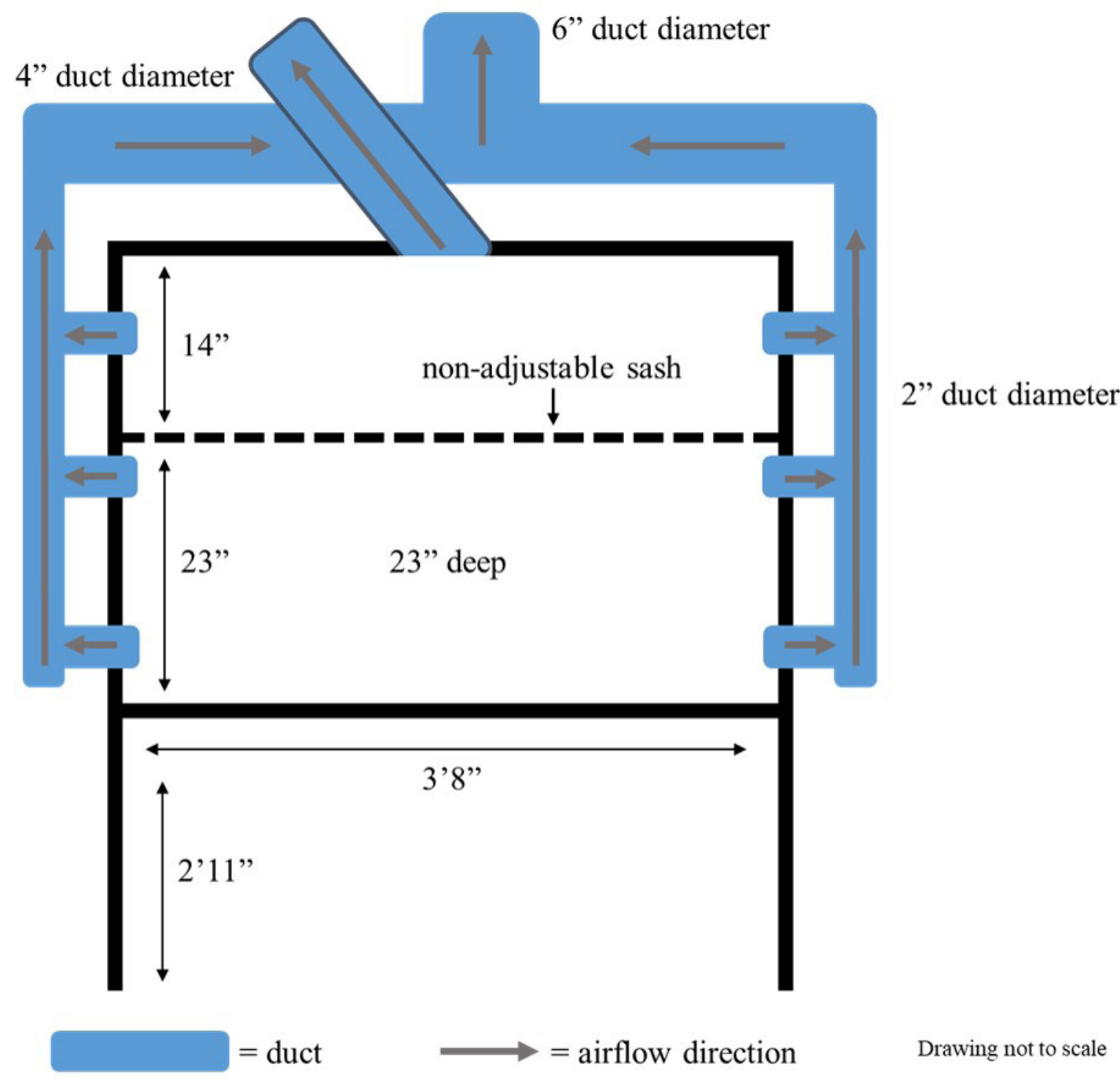

Figure 3. Diagram depicting the dimensions and configuration of the handmade hood in the printing department. The sash is not adjustable and there are two separate local exhaust systems connected to it. This hood is pictured in Figure 1. Figure by NIOSH. 


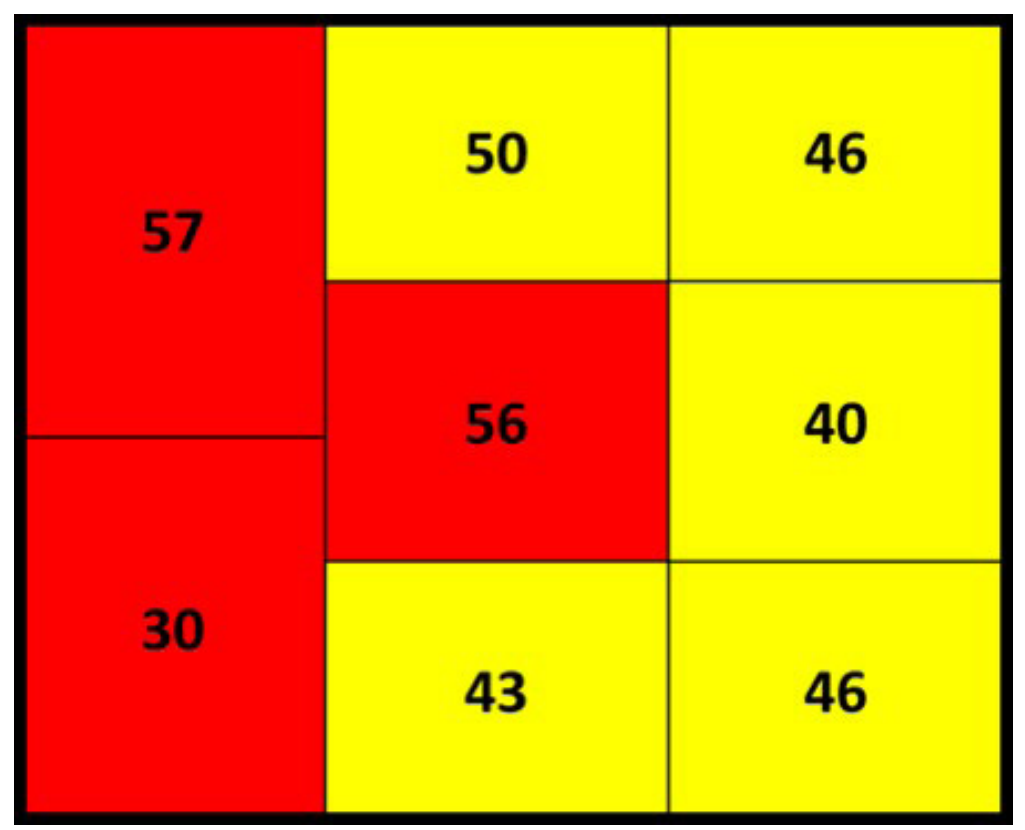

Figure 4. A diagram showing the face velocity measured at different points of the hood. There are only two measurements in the left column because the probe did not fit across the face of the hood three times and needed to be turned to measure the whole face. The red boxes indicate a greater than $20 \%$ difference from the average face velocity (46 feet per minute). All measurements were below OSHA and ANSI specifications. Figure by NIOSH.

\section{Employee Medical Interviews}

We held confidential medical interviews with 20 of 21 full-time employees who worked on the first- or second-shift in the production, molding, printing, or information technology departments. At the time of our visit, there were 87 full-time employees in all departments at the company. Eighty percent of interviewed employees spoke English as their primary language.

Employees reported working for the company an average of 12.6 years and at the facility an average of 10.3 years (range: $1.5-14$ years). Average age was 46 years old (range: $22-75$ ), and five were female. Employees usually worked an average of 41 hours a week (includes occasional overtime), Monday through Friday and occasionally Saturday.

Employees were asked to respond "yes" or "no" to a question regarding exposures to solvents (i.e., VOCs). Seven employees reported working with solvents including acetone, lacquer, resin, screen wash, printing inks, photographic fixer and developer, and/or adhesives. These employees usually worked with these solvents multiple times a day, but it depended on the scheduled work for the day. Short-term tasks using solvents included dispensing into containers/loading solvents into a machine, cleaning up and/or disposing of solvents.

Inhalation, ingestion, and transdermal are the routes of exposure to organic solvents; however, inhalation of solvent vapor is the most important route in occupational exposure. Dermal absorption is important when liquid solvents come into direct contact with the skin and this route is often overlooked [Xiao and Levin 2000]. U.S. workers are exposed to organic solvents that are used in such products as paints, varnishes, lacquers, adhesives, 
glues, and degreasing/cleaning agents, and in the production of dyes, polymers, plastics, textiles, printing inks, agricultural products, and pharmaceuticals [NIOSH 2017]. Some of the organic solvents that we sampled in the air and used in this facility included naphthalene, ethylbenzene, xylene, 2-butoxyethanol, and trimethylbenzene; the sampling did not detect any of these solvents in the air. These chemicals are ingredients found in the screen wash.

Four of the seven employees who worked with solvents reported wearing powder-free latex gloves and three employees reported wearing nitrile gloves. No employees reported wearing eye protection (safety glasses). Five of seven employees who worked with solvents reported occasionally noticing lacquer, ink, or acetone on uncovered parts of their body, especially their arms, hands, and/or face. All employees who worked with solvents reported they received training about solvent exposure when first hired, but were not offered periodic onsite or off-site refresher training. They reportedly received some basic information pertaining to certain job tasks.

When asked a specific question regarding changes in job tasks, procedures, and products used in the last year most employees reported that the products and procedures had been consistent. However, one employee reported that ventilation was better, and two employees reported job duty changes.

Employees were asked to respond "yes" or "no" to questions regarding symptoms. Eight employees reported no work-related symptoms. The most commonly reported symptoms by employees were lightheadedness, headache, and dizziness in the last 6 months (prominent in the afternoon during their shift). However, many of those employees reported their symptoms subsided and they were not currently experiencing any symptoms; some attributed the resolution of symptoms to improved ventilation. Many of those employees also reported a history of seasonal allergies (allergic rhinitis). VOCs could cause symptoms similar to those of seasonal allergies. Other common symptoms reported by employees included eye, nose, and throat irritation and/or dryness, sinusitis, and/or dry skin (some employees attributed this to frequent handwashing, dry environment, and touching solvents). Headaches, dizziness, lightheadedness, drowsiness, nausea, and eye and respiratory irritation have been associated with acute exposure to VOCs; symptoms go away with cessation of exposure [New York Department of Health 2018]. Because many of these symptoms are common and may be due to other causes, it is important for those who continue to experience them be evaluated by an occupational medicine physician to determine work-relatedness.

Thirteen employees reported a transient (e.g., once a day, 2-3 times a week or month) unpleasant odor or taste in mouth in the last 6 months. Three of those employees reported this odor or taste in mouth occurred between 2-4 months ago, and two of these employees reported this odor was stronger later in the work shift. Employees described the odor as a burning smell from molding machinery, lacquer, or plastic; diesel-like smell from fulfillment; alcohol-like smell from lacquer, screen wash, printing inks, other chemicals, and/ or plate developer. According to the SDSs, the screen printing inks and screen wash has a characteristic or typical odor and the resin and lacquer has a mild odor.

We asked employees an open-ended question regarding what, if any, health or safety concerns they had about their work. Eleven of twenty employees reported at least one safety and/or health concern. The main safety and health concerns reported by employees 
included inadequate safety and health training, issues with communication, ventilation and temperature changes, air circulation, odors, and/or potential long-term exposures to solvents. Two employees reported the company had made ventilation improvements, communication was sufficient, and/or that there were no language barriers between co-workers.

\section{Document Review}

\section{Medical Records Review}

We reviewed medical records for one employee who had seen multiple healthcare providers for symptoms that the employee thought were related to workplace exposures. We agreed with the healthcare providers' diagnosis of "possible reaction to chemicals (lacquer fumes)." The medical records documented work exposures as possible causes or contributors to symptoms.

\section{Logs of Injuries and IIInesses}

The Worker's Compensation Incident Report Log for the period covering 2013-2017 included 36 incidents in all departments and 14 of those incidents were in the departments we focused on. The majority of the incidents were musculoskeletal-related. There were two incidents in 2014 related to chemical exposure. These included chemical reaction to fumes and lacquers and possible chronic chemical exposure.

There was an OSHA Form 300A from 2013 on the wall during our visit. However, the company could not locate the accompanying 2013 OSHA 300 Log and was not preparing or maintaining OSHA 300 Logs from 2013-2017.

\section{Workplace Observations}

We observed limited use of PPE (e.g., safety glasses, protective gloves) by employees during our visit. Employees in the molding department were only provided powder-free latex gloves, which do not provide adequate protection from chemical exposures. The printing department had nitrile gloves. Employees reported not having training related to handling chemicals or the proper use of PPE. We observed food and drink in the molding and printing departments. There was a miniature refrigerator and microwave inside the molding department. Personal eye wash bottles were expired and some first aid kits were low on supplies. There were halfface elastomeric respirators that had been purchased in the past for the molding department. These respirators were not stored or cleaned properly. No employees or managers could recall any training on proper use and care for the respirators. The respirators were purchased under previous ownership and are not worn by any current employees. There is no respiratory protection program. The large book of SDSs was in need of updating. It was missing tabs to easily locate chemicals. There were unopened envelopes stuck in the back of the book from chemical companies. These envelopes likely contained updated SDSs. We observed containers of flammable chemicals being stored outside of flammable storage cabinets as well as inside other types of cabinets (e.g., filing cabinets in the printing department). There was no ventilation (e.g., a ventilation hood to use screen wash inside) available in the upstairs printing area. There was no clear method for transferring screen wash from the bulk shipping containers 
into the smaller plunger dispensers. The company did not offer to employees potentially exposed to solvents periodic onsite or off-site refresher hazard communication training on safe procedures, potential health effects, and how to reduce and prevent exposures.

\section{Conclusions}

We evaluated chemical exposures during optical media production. We did not detect naphthalene, ethylbenzene, xylene, 2-butoxyethanol, or trimethylbenzene in any of the samples. We observed a need for improved hazard communication, training, and availability of appropriate PPE. We also observed inconsistent and improper use of PPE. The transient unpleasant odor or taste in mouth reported in the last 6 months by many employees is consistent with exposure to some of the chemicals. Some employees reported symptoms in the last 6 months that may be due to chemical exposure or other causes. However, many of those employees reported their symptoms have recently subsided; some employees attributed the resolution of symptoms to improved ventilation.

\section{Recommendations}

On the basis of our findings, we recommend the actions listed below. We encourage the optical media production company to use a labor-management health and safety committee or working group to discuss our recommendations and develop an action plan. Those involved in the work can best set priorities and assess the feasibility of our recommendations for the specific situation at the company.

Our recommendations are based on an approach known as the hierarchy of controls (Appendix A). This approach groups actions by their likely effectiveness in reducing or removing hazards. In most cases, the preferred approach is to eliminate hazardous materials or processes and install engineering controls to reduce exposure or shield employees. Until such controls are in place, or if they are not effective or feasible, administrative measures and personal protective equipment may be needed.

\section{Engineering Controls}

Engineering controls reduce employees' exposures by removing the hazard from the process or by placing a barrier between the hazard and the employee. Engineering controls protect employees effectively without placing primary responsibility of implementation on the employee.

1. Consult with a ventilation engineer to investigate the source of, and to identify a way to prevent liquid from forming in the ductwork between the molding and printing departments.

2. Replace the handmade hood with one that complies with the OSHA standard 29 CFR 1910.1450 and ANSI standard Z9.5. The OSHA standard requires a "relatively uniform airflow" that is between 60 and 110 feet per minute. The ANSI standard recommends an average face velocity between 80 and 120 feet per minute and no face velocity measurement more than $20 \%$ different from the average velocity. The fan used to power the hood should be one that is appropriate to use in a chemical environment.

3. Acquire a means for mechanical transfer of the screen wash from bulk shipping 
containers into the smaller plunger cans. One option could be to use a manually operated flammable liquid drum pump. Ideally, the transfer should occur within a hood to prevent unnecessary exposure to the screen wash.

\section{Administrative Controls}

The term administrative controls refers to employer-dictated work practices and policies to reduce or prevent hazardous exposures. Their effectiveness depends on employer commitment and employee acceptance. Regular monitoring and reinforcement are necessary to ensure that policies and procedures are followed consistently.

1. Form a health and safety committee or working group to discuss our recommendations and develop an action plan. The group should be composed of an equal number of employer and employee representatives. A safety committee can help improve communication between management and employees. This group could organize fire drills and other emergency plans and set goals and track progress towards implementing changes in the workplace.

2. Prepare and maintain complete, descriptive, and accurate injury and illness information on OSHA Logs. Use injury and illness cases recorded on OSHA Logs and other incident reporting logs to look for trends in type of injury or illness over time and by job title or work area to target interventions. Companies are required to post $300 \mathrm{~A}$ in the workplace and replace it annually with a new 300A form per the OSHA recordkeeping rule [29 CFR 1904.35]. OSHA has a guide for filling out these forms https://www.osha.gov/recordkeeping/new-osha300form1-1-04.pdf.

3. Prohibit food and drink inside the molding and printing departments. Remove the miniature refrigerator and microwave from the molding department. Post signs in English and Spanish on the doors indicating no food or drink allowed in these areas. Communicate with all employees and contractors about the importance of not eating and drinking around chemicals in the workplace.

4. Wash hands and exposed areas of the body with water and soap after contact with chemicals, after removing gloves and before eating, smoking, or using the restrooms.

5. Inspect and replenish all first aid kits, spill kits, burn kits, eye wash stations, fire extinguishers, and any other emergency response equipment frequently. We noticed expired eye wash stations and first aid kits that were low on supplies.

6. Improve hazard communication within the workplace, following the OSHA Hazard Communication Standard [29 CFR 1910.1200]. OSHA provides detailed information about SDSs, training, and labeling requirements https://www.osha.gov/dsg/hazcom/. Contact your OSHA area office if you require assistance meeting regulatory requirements - https://www.osha.gov/html/RAmap.html.

- Many SDSs were out of date. The large book of SDSs in the hallway near the vending machines was missing tabs throughout the book to identify where individual SDSs were located. Additionally, there were many unopened letters in the back of the binder that were likely updated SDSs sent from chemical companies. 
- Ensure all employees potentially exposed to chemicals are trained and knowledgeable about chemical hazards at the workplace and how these exposures affect their health. Employees should know how to prevent exposures when working with and around the chemicals. This training should be completed when hired and periodically as refresher training after that. Training should be offered in English and Spanish to make sure employees are comfortable and understand the material. Training should include contractors who handle the waste produced in the molding and printing departments.

7. Improve chemical storage in the molding and printing departments. We observed containers of flammable chemicals being stored outside of flammable storage cabinets as well as inside other types of cabinets (e.g., filing cabinets in the printing department). The SDSs contain information about handling and storage of the chemicals. For example:

- Screen wash should be stored in a flammable storage cabinet.

- Resin and lacquer should be stored away from sunlight and with spillage containment in case of a leak in the original containers.

- All secondary containers should be properly labeled. Acetone and isopropanol had appropriate secondary containers, but other chemicals (e.g., screen wash) were in unlabeled or poorly labeled secondary containment.

8. Investigate the strong chemical smells in the bathrooms. Check with the cleaning contractor and ensure that contractors are using cleaning chemicals according to the manufacturers' recommendations. Check to see whether the automatic air fresheners in the bathrooms can be set to spray less frequently. Encourage employees to avoid using their own air freshening products in the bathrooms at work.

9. Encourage employees to report work-related health and safety concerns to their supervisors; employees with persistent symptoms should seek medical attention from a healthcare provider who is knowledgeable in occupational medicine.

\section{Personal Protective Equipment}

Personal protective equipment is the least effective means for controlling hazardous exposures. Proper use of personal protective equipment requires a comprehensive program and a high level of employee involvement and commitment. The right personal protective equipment must be chosen for each hazard. Supporting programs such as training, changeout schedules, and medical assessment may be needed. Personal protective equipment should not be the sole method for controlling hazardous exposures. Rather, personal protective equipment should be used until effective engineering and administrative controls are in place.

1. Require safety glasses be worn by all employees, contractors (i.e., cleaning contractors, temporary agency staff), and visitors in the molding and printing departments. Safety glasses should be worn at all times, even when the machines are powered off. Post signs on doors in English and Spanish to indicate eye protection requirement. Provide safety glasses at all entrances to these areas to ensure compliance. Communicate with all employees and contractors about the new safety requirement. 
2. Replace latex gloves with thick $(>0.5 \mathrm{~mm})$ nitrile gloves. Employees should wear them when handling screen wash, resin, lacquer, and other solvents (e.g., acetone) and remove and dispose of gloves after a single use. They should also be instructed to:

- Remove gloves before leaving the molding and printing departments.

- Change gloves when there is a change in appearance (e.g., color change, flexibility, visibly contaminated).

- Never reuse gloves once they have been removed.

3. Encourage all employees in the molding and printing departments to wear nitrile gloves and long sleeves to prevent unnecessary dermal exposures to solvents.

4. Dispose of all respirators and cartridges that were purchased to wear in the molding department while working with the resin and lacquer. These respirators have not been properly cleaned, stored and maintained and are likely approaching their expiration dates.

5. Ensure that all respirators are certified by NIOSH. The certified equipment list maintained by NIOSH can be found here: https://www.cdc.gov/niosh/npptl/topics/respirators/cel/default.html.

6. Ensure that you comply with the voluntary use provisions of the OSHA respiratory protection standard (29 CFR 1910.134) if you plan to allow employees to wear filtering facepiece respirators during optical media production. We found no evidence that respiratory protection should be required. 


\section{Appendix A: Occupational Exposure Limits and Health Effects}

NIOSH investigators refer to mandatory (legally enforceable) and recommended OELs for chemical, physical, and biological agents when evaluating workplace hazards. OELs have been developed by federal agencies and safety and health organizations to prevent adverse health effects from workplace exposures. Generally, OELs suggest levels of exposure that most employees may be exposed to for up to 10 hours per day, 40 hours per week, for a working lifetime, without experiencing adverse health effects. However, not all employees will be protected if their exposures are maintained below these levels. Some may have adverse health effects because of individual susceptibility, a pre-existing medical condition, or a hypersensitivity (allergy). In addition, some hazardous substances act in combination with other exposures, with the general environment, or with medications or personal habits of the employee to produce adverse health effects. Most OELs address airborne exposures, but some substances can be absorbed directly through the skin and mucous membranes.

Most OELs are expressed as a time-weighted average (TWA) exposure. A TWA refers to the average exposure during a normal 8- to 10-hour workday. Some chemical substances and physical agents have recommended short-term exposure limit or ceiling values. Unless otherwise noted, the short-term exposure limit is a 15-minute TWA exposure. It should not be exceeded at any time during a workday. The ceiling limit should not be exceeded at any time.

In the United States, OELs have been established by federal agencies, professional organizations, state and local governments, and other entities. Some OELs are legally enforceable limits; others are recommendations.

- The U.S. Department of Labor OSHA permissible exposure limits (29 CFR 1910 [general industry]; 29 CFR 1926 [construction industry]; and 29 CFR 1917 [maritime industry]) are legal limits. These limits are enforceable in workplaces covered under the Occupational Safety and Health Act of 1970.

- NIOSH RELs are recommendations based on a critical review of the scientific and technical information and the adequacy of methods to identify and control the hazard. NIOSH RELs are published in the NIOSH Pocket Guide to Chemical Hazards [NIOSH 2010]. NIOSH also recommends risk management practices (e.g., engineering controls, safe work practices, employee education/training, personal protective equipment, and exposure and medical monitoring) to minimize the risk of exposure and adverse health effects.

- Another set of OELs commonly used and cited in the United States is the ACGIH TLVs. The TLVs are developed by committee members of this professional organization from a review of the published, peer-reviewed literature. TLVs are not consensus standards. They are considered voluntary exposure guidelines for use by industrial hygienists and others trained in this discipline "to assist in the control of health hazards" [ACGIH 2018]. 
Outside the United States, OELs have been established by various agencies and organizations and include legal and recommended limits. The Institut für Arbeitsschutz der Deutschen Gesetzlichen Unfallversicherung (Institute for Occupational Safety and Health of the German Social Accident Insurance) maintains a database of international OELs from European Union member states, Canada (Québec), Japan, Switzerland, and the United States. The database, available at http://www.dguv.de/ifa/GESTIS/GESTIS-Internationale-Grenzwerte-fürchemische-Substanzen-limit-values-for-chemical-agents/index-2.jsp, contains international limits for more than 2,000 hazardous substances and is updated periodically.

OSHA requires an employer to furnish employees a place of employment free from recognized hazards that cause or are likely to cause death or serious physical harm [Occupational Safety and Health Act of 1970 (Public Law 91-596, sec. 5(a)(1))]. This is true in the absence of a specific OEL. It also is important to keep in mind that OELs may not reflect current health-based information.

When multiple OELs exist for a substance or agent, NIOSH investigators generally encourage employers to use the lowest OEL when making risk assessment and risk management decisions. NIOSH investigators also encourage use of the hierarchy of controls approach to eliminate or minimize workplace hazards. This includes, in order of preference, the use of (1) substitution or elimination of the hazardous agent, (2) engineering controls (e.g., local exhaust ventilation, process enclosure, dilution ventilation), (3) administrative controls (e.g., limiting time of exposure, employee training, work practice changes, medical surveillance), and (4) personal protective equipment (e.g., respiratory protection, gloves, eye protection, hearing protection). Control banding, a qualitative risk assessment and risk management tool, is a complementary approach to protecting employee health. Control banding focuses on how broad categories of risk should be managed. Information on control banding is available at http://www.cdc.gov/niosh/topics/ctrlbanding/. This approach can be applied in situations where OELs have not been established or can be used to supplement existing OELs. 


\section{References}

ACGIH [2018]. 2018 TLVs ${ }^{\circledR}$ and BEIs ${ }^{\circledR}$ : threshold limit values for chemical substances and physical agents and biological exposure indices. Cincinnati, OH: American Conference of Governmental Industrial Hygienists.

CFR. Code of Federal Regulations. Washington, DC: U.S. Government Printing Office, Office of the Federal Register.

New York Department of Health [2018]. Volatile organic compounds in commonly used products. https://www.health.ny.gov/publications/6513.

NIOSH [2010]. NIOSH pocket guide to chemical hazards. Cincinnati, OH: U.S. Department of Health and Human Services, Centers for Disease Control and Prevention, National Institute for Occupational Safety and Health, DHHS (NIOSH) Publication No. 2010-168c, http://www.cdc.gov/niosh/npg/.

NIOSH [2017]. Workplace safety and health topics - organic solvents. https://www.cdc.gov/niosh/topics/organsolv/.

NIOSH [2018]. NIOSH manual of analytical methods (NMAM). 5th ed. O'Connor PF, Ashley K, eds. Cincinnati, OH: U.S. Department of Health and Human Services, Centers for Disease Control and Prevention, National Institute for Occupational Safety and Health, DHHS (NIOSH) Publication No. 2014-151, http://www.cdc.gov/niosh/nmam.

Wallace LA [1986]. Personal exposures, indoor and outdoor air concentrations and exhaled breath concentrations of selected volatile organic compounds measured for 600 residents of New Jersey, North Dakota, North Carolina, and California. Toxicol Environ Chem 12(3-4):215-236, https://doi.org/10.1080/02772248609357160.

Wallace LA [1991]. Comparison of risks from outdoor and indoor exposure to toxic chemicals. Environ Health Perspect 95:7-13, https://doi.org/10.2307/3431099.

Wallace LA, Pellizzari ED, Leaderer B, Zelon H, Sheldon L [1987]. Emissions of volatile organic compounds from building materials and consumer products. Atmos Environ 21(2):385-393, https://doi.org/10.1016/0004-6981(87)90017-5.

Xiao JQ, Levin SM [2000]. The diagnosis and management of solvent-related disorders. Am J Ind Med 37(1):44-61, https://doi.org/10.1002/(SICI)1097-0274(200001)37:1<44::AID-AJIM5>3.0.CO;2-K. 
Keywords: North American Industry Classification System (NAICS) 334614

(Software and Other Prerecorded Compact Disc, Tape, and Record Reproducing); Illinois;

Optical Media Production; Odor; Molding; Printing; Naphthalene, Ethylbenzene, Xylene, 2Butoxyethanol, Trimethylbenzene 
This page left intentionally blank 
The Health Hazard Evaluation Program investigates possible health hazards in the workplace under the authority of the Occupational Safety and Health Act of 1970 (29 U.S.C. § 669(a) (6)). The Health Hazard Evaluation Program also provides, upon request, technical assistance to federal, state, and local agencies to investigate occupational health hazards and to prevent occupational disease or injury. Regulations guiding the Program can be found in Title 42, Code of Federal Regulations, Part 85; Requests for Health Hazard Evaluations (42 CFR Part 85).

\section{Disclaimer}

The recommendations in this report are made on the basis of the findings at the workplace evaluated and may not be applicable to other workplaces.

Mention of any company or product in this report does not constitute endorsement by NIOSH.

Citations to Web sites external to NIOSH do not constitute NIOSH endorsement of the sponsoring organizations or their programs or products. NIOSH is not responsible for the content of these Web sites. All Web addresses referenced in this document were accessible as of the publication date.

\section{Acknowledgments}

Analytical Support: Maxxam Analytics

Desktop Publisher: Shawna Watts

Industrial Hygiene Field Assistance: Catherine Beaucham, Donnie Booher

Logistics: Donnie Booher, Kevin Moore

Medical Field Assistance: Guadalupe Gomez

\section{Availability of Report}

Copies of this report have been sent to the employer and employees at the facility. The state and local health department and the Occupational Safety and Health Administration Regional Office have also received a copy. This report is not copyrighted and may be freely reproduced.

\section{Recommended citation for this report:}

NIOSH [2018]. Evaluation of chemical exposures during optical media production. By Grant MP, Musolin K. Cincinnati, OH: U.S. Department of Health and Human Services, Centers for Disease Control and Prevention, National Institute for Occupational Safety and Health, Health Hazard Evaluation Report 2017-0078-3323, https://www.cdc.gov/niosh/hhe/reports/pdfs/2017-0078-3323.pdf. 
Delivering on the Nation's promise:

Promoting productive workplaces through safety and health research

To receive NIOSH documents or more information about occupational safety and health topics, please contact NIOSH:

Telephone: 1-800-CDC-INFO (1-800-232-4636)

TTY: 1-888-232-6348

CDC INFO: www.cdc.gov/info

or visit the NIOSH Web site at www.cdc.gov/niosh

For a monthly update on news at $\mathrm{NIOSH}$, subscribe to

$\mathrm{NIOSH}$ eNews by visiting www.cdc.gov/niosh/eNews. 